\title{
Lidil
}

Revue de linguistique et de didactique des langues

47 | 2013

Le verbe pour exprimer le temps

\section{Quelle expression du temps pour les formes non fléchies du verbe ? Examen de quelques manuels de CM1}

Frédéric Torterat

\section{(2) OpenEdition \\ Journals}

Édition électronique

URL : http://journals.openedition.org/lidil/3266

DOI : $10.4000 /$ lidil.3266

ISSN : 1960-6052

Éditeur

UGA Éditions/Université Grenoble Alpes

\section{Édition imprimée}

Date de publication : 31 mai 2013

Pagination : 81-98

ISBN : 978-2-84310-247-9

ISSN : 1146-6480

Référence électronique

Frédéric Torterat, «Quelle expression du temps pour les formes non fléchies du verbe ? Examen de quelques manuels de CM1 », Lidil [En ligne], 47 | 2013, mis en ligne le 01 décembre 2014, consulté le 05 mai 2019. URL : http://journals.openedition.org/lidil/3266 ; DOI : 10.4000/lidil.3266 


\title{
Quelle expression du temps pour les formes non fléchies du verbe? Examen de quelques manuels de CM1
}

\author{
Frédéric Torterat*
}

\begin{abstract}
RÉSUMÉ
Si les temporalités exprimées par les formes verbales fléchies ont fait l'objet d'une recherche abondante et diversifiée, y compris sur les démarches les plus à même d'en faciliter l'apprentissage, les approches de la contribution des formes non fléchies à l'expression du temps sont beaucoup plus hésitantes. L'une des difficultés revient au fait que les participes, l'infinitif et le gérondif se rattachent généralement, dans leur expression du temps, à d'autres éléments du contexte (comme les verbes fléchis de leur entourage, ainsi que des circonstants). De tels mécanismes étant pris en compte jusque dans les manuels scolaires, leur existence nous conduit à nuancer les approches du temps verbal qui s'en tiennent strictement à des questions de sémantique lexicale et/ou de morphologie flexionnelle. Pour démontrer les faits concrètement, nous procédons ici à une analyse comparative de manuels de CM1 parus entre 2009 et 2012, en envisageant dans quelle mesure ces ouvrages incorporent l'infinitif, les participes et éventuellement le gérondif à la (re)présentation et à la manipulation du temps verbal.
\end{abstract}

\begin{abstract}
Temporality expressed by finite verbs has received a great deal of attention in theoretical linguistic works as well as in pedagogical studies. In contrast, approaches to the way non-finite verbs contribute to the expression of temporality are more tentative. One of the main difficulties in the analysis of participles, infinitives and gerunds is that they are generally linked to other elements of the context, such as finite verbs and adjuncts. Such mechanisms are repeatedly identified in school textbooks, and their existence leads us to reconsider grammatical approaches to verb tense-for these approaches are too strictly
\end{abstract}

* Université de Nice Sophia-Antipolis, IUFM Célestin Freinet (EA 6308). 
anchored in lexical semantics and/or inflectional morphology. To illustrate these facts, this paper carries out a comparative analysis of 4th Grade textbooks published between 2009 and 2012; it examines to what extent such books incorporate infinitives, participles and gerunds to the (re)presentation and manipulation of verbal tense.

La temporalité exprimée par les formes fléchies du verbe a fait l'objet d'une recherche abondamment documentée, et ce dans de multiples domaines. Parmi ces domaines et depuis au moins les années 1980, la didactique de la grammaire, et notamment celle dite «de la conjugaison», ont apporté des réponses concrètes sur l'emploi et le réemploi, par les élèves, des «formes temporo-verbales» (David \& Renvoisé, 2010), mais aussi sur les supports pédagogiques les plus à même d'en faciliter l'apprentissage.

Concernant les formes non fléchies et sur cette question du temps en particulier, les éclairages sont beaucoup plus indirects. Cela tient principalement au fait que celles-ci apparaissent avant tout comme non marquées, autrement dit, pour ce qui nous occupe ici, atemporelles. Pour autant, même si les participes (passé, présent), l'infinitif et le gérondif ne disposent d'aucune marque flexionnelle, ces derniers participent des représentations que les locuteurs se font du temps effectivement signifié, ainsi que de l'aspect (l'infinitif, le participe présent et le gérondif peuvent apparaitre, notamment, aux formes simple et composée). L'une des difficultés, en termes de construction temporelle, revient à ce que ces formes verbales se rattachent généralement, dans leur contribution à cette expression, à d'autres éléments du contexte (comme les verbes fléchis de leur entourage, ainsi que des circonstants). De tels mécanismes étant pris en compte jusque dans les manuels scolaires, ils nous conduisent à nuancer les classements et les approches grammaticales du temps verbal qui s'en tiennent à une stricte «dérivation morphologique» des formes fléchies du verbe (Masseron, 1998), délaissant par moments un peu vite la contribution des formes non fléchies (dans leur ensemble) à l'expression du temps. Pour démontrer les faits concrètement, nous exemplifierons notre propos à partir d'une analyse comparative d'ouvrages pédagogiques postérieurs à 2008 , en envisageant dans quelle mesure ces supports incorporent l'infinitif, les participes et éventuellement le gérondif à la (re)présentation et à la manipulation du temps verbal. Après avoir défini les principaux domaines d'analyse envisageables sur ces questions, nous déterminerons comment de tels contenus peuvent être proprement didactisés, en confrontant l'approche qu'en font quelques manuels de CM1 parus entre 2009 et 2012. 


\section{Premiers éléments}

La plupart des ouvrages de grammaire qui pratiquent une approche descriptive du verbe présentent les caractéristiques de la catégorie en distinguant les «modes personnels » aux marques temporelles variées, des «modes impersonnels », qui sont aussi définis, dans bien des cas, comme «intemporels » (Arrivé, Gadet \& Galmiche, 1986; Riegel, Pellat \& Rioul, 1999; Eluerd, 2011). En l'occurrence pour le français, l'infinitif et le gérondif, de même que les participes, occupent une place à part, y compris quand ils sont abordés avec des «paramètres » similaires à ceux qui sont utilisés pour décrire les formes dites «conjuguées ». Même quand le gérondif, l'infinitif et le participe «sont répertoriés à égalité avec les autres formes verbales» (Meleuc \& Fauchart, 1999, p. 81), il n'est généralement question, pour eux, d'aucune valeur temporelle qu'il leur appartiendrait de manifester. Les classements qui en sont déduits donnent souvent l'impression d'une répartition discutable, dont il est difficile de concevoir l'opportunité didactique au regard du système verbal dans son ensemble, d'autant que les tentatives d'éclaircissement du «"monstrueux" problème de la conjugaison française », suivant la formule de Germain \& Séguin (1998), sont pour ainsi dire tout entières occupées par le «système flexionnel» (id., p. 74) et par ses régularités (Dressler, 1997; Bonami \& Boyer, 2008). Ces tendances se vérifient en particulier pour ce qui touche à l'expression du temps verbal, d'autant que, pour reprendre Cogis (2005, p. 116), avec le verbe,

tout est compliqué : caractère abstrait des notions sous-jacentes, spécialisation rigoureuse de graphèmes polyvalents et plurifonctionnels, multiplicité des agencements, jeu d'alternance entre segments phoniques et donc graphiques, ou segments graphiques seulement, intervention significative d'un élément.

Le cas de l'infinitif est à ce titre révélateur : avec cette forme non fléchie, la construction de la temporalité s'opère le plus souvent en lien avec un verbe fléchi plus ou moins proche (ainsi dans des constructions co-verbales ou subordinatives : ils la laissent s'amuser un peu / je l'ai vu entrer dans la classe). Cela étant, l'infinitif a aussi la capacité d'être employé pour lui-même : outre des formules figées comme à dire vrai, ou encore des cas spécifiques tel que l'emploi dit «de narration» (et un tel de parler), l'infinitif peut intervenir comme noyau principal d'assertions (ça alors, parler ainsi!), de questions (comment dire?) et d'injonctions (ne pas claquer la porte), où il exprime des temporalités diverses. L'un des enjeux didactiques est par conséquent de faire saisir 
aux élèves, dès l'école élémentaire, en quoi l'infinitif a la capacité de signifier, outre un présent et un passé, plusieurs «visions » temporelles (Charaudeau, 1992) ${ }^{1}$. Ces données sont effectivement un élément clé de la compréhension qu'ont les élèves, de L1 comme de L2, des temporalités représentées dans l'organisation discursive (et textuelle). La didactisation de tels contenus d'apprentissage passe donc par la nécessaire combinaison d'une approche morphologique (formes simples et composées, régularités graphiques) et d'une utile contextualisation des emplois des formes verbales (dont l'infinitif), dans des ensembles qui ne se limitent ni au groupe verbal, ni à la phrase.

Le gérondif, pour sa part, peut être envisagé, comme l'infinitif, à travers l'expression de ce qui est en voie d'accomplissement comme forme simple d'une part (en disant cela), et de ce qui est accompli d'autre part, quand il intervient comme composé (en ayant dit cela). Outre ces significations d'aspect, le gérondif convoque lui aussi une temporalité liée au verbe fléchi auquel il est rattaché (elle entre [est entrée lentrera] en faisant du bruit). Ces particularités supposent de ne pas faire automatiquement coïncider l'emploi du gérondif avec la représentation de la seule simultanéité, d'autant qu'un tel automatisme présente le défaut incontestable d'en marginaliser la forme composée (Halmøy, 2003; Kleiber, 2007). Cela s'applique aussi aux configurations où le gérondif inscrit le procès dans l'antériorité, la postériorité ou une éventualité temporellement indéterminée (en ayant dit [en disant] cela, vous vous compromettriez/ compromettez).

Pour ce qui concerne les autres formes non fléchies, il apparait que le participe passé est rattaché temporellement à des éléments du cotexte, en ceci qu'il lui est très difficile d'exprimer des temporalités variées par lui-même ou à travers des relations distantes. Qui plus est, le participe passé ne dispose que de sujets «obliques», pour reprendre le terme de Lazard (1985). De telles contraintes ne pèsent pas sur le participe présent, qui, en plus de la possibilité d'une forme composée (les élèves produisant [ayant produit] un écrit), dispose dans bien des cas d'un sujet propre et s'assortit de diverses «valeurs» temporelles.

1. Pour ce que recommandent à ce titre les Programmes français de l'École primaire de 2008, voir infra (2.2.). Les «visions» énumérées par Charaudeau reformulent en partie la question de l'aspect, que nous définirons, avec Tournadre (2004, p. 17), comme «la position adoptée par le locuteurénonciateur», «la perspective choisie par ce dernier pour envisager la situation ou l'évènement qu'il souhaite évoquer». 
Quelle que soit la démarche mise en œuvre en contexte d'apprentissage, la perspective du rattachement de l'infinitif, des participes et du gérondif à d'autres éléments discursifs (dont des verbes fléchis) peut s'avérer particulièrement efficace sur le plan didactique, car à travers ces rapprochements, de multiples significations temporelles (ré)apparaissent. Celles-ci se concrétisent pédagogiquement dans les questions de savoir sur quoi s'appuie le verbe, ou plus généralement ce qui, parmi les éléments qui l'entourent, l'amène à contribuer à l'expression du temps, comme nous l'envisagerons ci-après à travers ce qu'en font, en pratique, quelques manuels scolaires. La démarche consiste à amener les élèves à saisir et à désigner par eux-mêmes toutes les formes verbales qui permettent de construire le temps, et cela sans s'arrêter aux verbes conjugués. D'ailleurs et comme l'indiquent David \& Renvoisé (2010, p. 72) :

L'objectif n'est sans doute pas de connaitre la totalité des paradigmes de conjugaison de leurs répertoires (Bescherelle ou autres), mais plutôt de savoir les utiliser en combinaison ou en alternance avec d'autres supports, comme les manuels, les dictionnaires, les correcteurs informatiques, etc.

Ces complémentarités profitent tout particulièrement au verbe, dont la première caractéristique revient au fait qu'il «constitue le domaine par excellence de la variation» (Lusetti, 2008, p. 109). Au demeurant et quelles que soient les pratiques pédagogiques à l'œuvre, il s'agit bien, comme l'écrit Meleuc (2005, p. 50), de s'inscrire «à l'inverse des approches linéaires, unifiantes, centrées sur le mot, qui donnent une image lisse du verbe, et dont le symbole pédagogique serait le tableau de conjugaison, avec comme pendant pratique la récitation du verbe ${ }^{2} \gg$. Nous y reviendrons dans notre conclusion, mais cela revient à dire de notre point de vue qu'une approche didactique du verbe a tout intérêt à prendre en compte les dimensions à la fois variationniste, mais aussi discursive de ce contenu d'apprentissage, en se refusant notamment à marginaliser des formes que les élèves utilisent et reconnaissent, mais qu'on laisse de côté sous des prétextes divers ${ }^{3}$.

2. Les italiques sont de l'auteur.

3. Meleuc (2005, p. 63) suggère dans cet esprit de partir par exemple d'un «genre textuel précis, un type discursif, et [de] regarder, par opposition, 


\section{Au regard des faits linguistiques}

\subsection{Sur quoi s'appuie la forme verbale : la piste du rattachement}

Concédons que les emplois des formes fléchies du verbe s'accompagnent d'indéniables récurrences, qui sont autant de facilitations possibles pour l'apprentissage : celles qu'implique l'indicatif, par exemple, donnent une représentation discursivement plus posée (si ce n'est factuelle) aux contenus que le subjonctif, lequel présente dans presque tous les cas les procès comme envisagés (en renvoyant notamment à des évènementialités présumées ou éventuelles). Le temps exprimé, par exemple pour le présent ou l'imparfait de l'indicatif, même s'il s'inscrit dans de multiples possibles, s'y construit de manière proprement plus accessible que dans les cas des participes, de l'infinitif et du gérondif. Ces tendances s'expliquent par ce qu'en dehors de leur construction, les formes non fléchies présentent une incomplétude de re qui les place en général dans les champs de l'apport complémentaire et/ou d'une prédication seconde (Havu, 2009; Torterat, 2012b). La question est donc : sur quoi s'appuient ces formes verbales, concrètement, pour contribuer à l'expression du temps?

En linguistique, la question du rattachement permet de cerner ce qui caractérise certaines constructions spécifiques, comme les appositions ou les coordinations «atypiques» par exemple. Dans un article à certains égards fondateur (2009), Deulofeu insiste sur le fait que, quelles que soient les constructions impliquées, «c'est la question du mode de rattachement qu'il faut aborder» (p. 235). Il en est ainsi pour les constituants détachés (tels les groupes de mots organisés autour de participes ou de gérondifs), vis-à-vis desquels le problème «n'est pas de se demander de quoi ces constituants sont détachés, mais comment ils sont rattachés au contexte» (p. 229). Même quand il s'agit d'éléments incidents au verbe principal ou à la phrase «hôte» (comme les infinitifs enchâssés), il est par conséquent tout «aussi approprié de dire que [tel] élément dépend de l'ensemble du contexte discursif ou du "paragraphe" qui le contient» (p. 240). Ces opérations s'appliquent indéniablement aux formes verbales, et c'est pourquoi, comme d'autres auteurs, nous aborderons le rattachement comme un mécanisme de relation entre un élément support et un ou plusieurs apport(s), avec le(s)quel(s) il

comment les formes sont utilisées dans le texte, comment on les reconnait à la lecture, comment on les dénomme» (cf. Dourojeanni \& Quet, 2007). 
forme un (sous-)ensemble en production ${ }^{4}$. Indiquons par ailleurs que la plupart des approches grammaticales contemporaines représentent le rattachement comme lié à la question de sa localité, ce qui le rend particulièrement efficace pour l'analyse. Deulofeu (2010) parle ainsi, pour les rattachements non locaux, de «portée sémantique large» (p. 360 $s q q$.), en «aval» comme en «amont du discours $»^{5}$. De notre point de vue, cette question du rattachement permet de cerner au plus près ce qui relie temporellement des formes non fléchies du verbe soit à un élément du cotexte, soit à un ou plusieurs éléments contextuels (éventuellement extraverbaux), comme le concèdent Gettrup (1977, p. 218) pour le gérondif, en parlant de «faits extralinguistiques », ou encore Martin (1971) pour l'infinitif, en parlant pour sa part de «contexte».

\subsection{Quel apport des manuels scolaires?}

Afin de montrer comment ces questions sont opportunément transposables en contexte didactique, en particulier pour ce qui concerne la construction du temps par les formes verbales non fléchies, nous comparerons, dans la section qui suit, les recours qu'en font quelques manuels scolaires de CM1 (pour des enfants de 9-10 ans), et surtout comment ces ouvrages les inscrivent, ou non, dans une représentation unificatrice et décloisonnée (formes conjuguées et non conjuguées) du temps verbal. Rappelons avant tout que dans la suite d'une reprécision générale des exigences académiques (en grammaire comme ailleurs) qui s'est affinée dans les années 2000, les Programmes (2008) de l'École primaire recommandent, pour les classes du cycle 3 de l'École primaire (CE2, CM1 et CM2), de faciliter la «compréhension» du système verbal en exerçant les élèves au «repérage dans un texte des temps simples et des temps composés de l'indicatif», ainsi que ce qui correspond à leur «formation» (de même que pour les autres modes, comme le «conditionnel» et l'impératif). Outre les conjugaisons elles-mêmes, parmi lesquelles apparaissent comme telles les participes (présent, passé) et

4. Les notions ici d'apport et de support ne renvoient pas à l'usage qu'en font Van Raemdonck \& Detaille (2011), lesquels y présentent une approche qui leur est spécifique.

5. Les termes ne sont évidemment pas les mêmes suivant les approches méthodologiques retenues. Les contiguïtés entre eux sont toutefois évidentes, par exemple chez Muller (2006), qui mentionne une «dépendance distendue» pour les rattachements non locaux (cf. Torterat, 2012a, 2012b). 
l'infinitif («présent»), il s'agit de pratiquer «une première approche de la valeur des temps verbaux et en particulier des temps du passé». Pour ce qui relève des autres formes non fléchies du verbe, le gérondif n'est pas cité, bien qu'il intervienne beaucoup plus couramment à l'oral et dans les productions écrites des élèves que le participe présent. Cela étant, tout porte à croire que la dénomination de participe présent, dans les Programmes, n'exclut pas complètement le gérondif et recouvre dans les faits les formes en -ant (dont les adjectifs verbaux). Cette imprécision n'est d'ailleurs pas l'apanage de ce type de document, et il est fréquent de relever un certain flottement (par ailleurs historiquement fondé) dans des articles par ailleurs très bien documentés.

Sur le plan didactique et comme nous l'avons brièvement suggéré en 2.1., la perspective du rattachement linguistique permet de ménager l'une des formes possibles de ce que Meleuc (2005, p. 56) appelle une «phase sérieuse d'observation des formes en contexte». Il nous semble donc opportun de considérer les types de soutien qu'apportent les manuels scolaires à cette démarche de relevé et de compréhension, d'autant que les manuels restent, en CM1 comme dans l'ensemble du cycle 3 de l'École primaire, des supports pédagogiques régulièrement sollicités pour l'apprentissage du système verbal. En effet, la représentation et l'exercice des formes verbales dans ces supports pédagogiques y font généralement l'objet de rubriques spécifiques, en rapport soit avec la «grammaire du verbe» (le verbe dans la phrase, le sujet, etc.), soit avec l'orthographe (accord sujet-verbe, participes passés, homophones grammaticaux), soit avec la conjugaison proprement dite ou diverses productions d'écrits. De nombreux travaux ont traité ces questions, en reprenant notamment les procédés auxquels recourent les manuels pour favoriser la prise en compte contexte discursif (ainsi que des genres textuels), dans la mesure où, comme le rappelle Lusetti (2008, p. 113), le contexte "permet également de construire la notion de verbe», y compris (et surtout) quand il est envisagé comme «un élément caractéristique de [...] la temporalité». Or, l'intégration des formes verbales non fléchies dans les séances prévues dans les manuels, quelquefois très abouties, est sensiblement plus allusive et presque toujours indirecte. Nous en donnerons davantage d'illustrations dans la section suivante, mais si nous prenons par exemple Mot de passe CM1 (Hachette, 2010), les formes non fléchies n'apparaissent pour elles-mêmes, dans les intitulés des rubriques (reportées dans le sommaire), qu'à travers l'infinitif et le participe passé : une démarche tout à fait représentative de ce qui s'établit généralement dans les manuels. Dans Mot de passe 
$C M 1$, si l'infinitif est envisagé proprement comme forme verbale, c'est en «conjugaison» et à travers la distinction qu'il permet avec le «verbe conjugué » (p. 32-33), ou en «orthographe» (p. 208-209) au titre de la distinction des homophones (infinitifs, participes passés et imparfaits). Le participe passé, de son côté, est abordé indirectement, en «conjugaison», à la faveur des «temps composés » (p. 122-123, 140-141, 152153 et 212-213 dans une moindre mesure), ainsi qu'en «orthographe » à l'occasion des accords (p. 118-119 et 166-167) ou de la distinction des homophones (p. 208-209).

Ces éléments déclaratifs présents dans les intitulés ne préfigurent toutefois en rien le recours effectif et concret que font les manuels des participes, de l'infinitif et éventuellement du gérondif, dans les séances consacrées aux apprentissages grammaticaux et même par ailleurs (en «écriture», par exemple). Ces formes verbales apparaissent en effet dans de multiples exercices liés notamment aux «types de phrases », à l' «utilisation des temps du passé», voire aux «marqueurs de temps». Elles vont même jusqu'à intervenir dans des manipulations consacrées à la «concordance des temps», laquelle fait d'ailleurs l'objet de représentations variées (voir sur ce point Sautot \& Lepoire-Duc, 2009). Pour ne citer que deux exemples de Mot de passe CM1, l'exercice 11 de la page 31 sur le «sujet du verbe» demande aux élèves de relever et de recopier des «infinitif[s] ou groupes[s] infinitif[s]» dans un texte narratif, et l'exercice 6 de la page 77 (sur les «mots invariables») les invite à remplacer un groupe gérondif («en faisant du bruit») ainsi qu'un groupe infinitif («sans me lasser»), tous deux employés en contexte, par des adverbes. Nous relevons des pratiques éditoriales similaires dans la plupart des manuels de CM1 : ainsi l'ouvrage Outils pour le Français (Magnard, 2008), qui insiste beaucoup sur la morphologie des temps verbaux, intègre-t-il l'infinitif dans de sommaires productions d'écrits (par exemple 8-9 page 59 et 7 page 61), mais aussi dans des manipulations diverses à partir de récits au passé. De même, le Français, étude de la langue CM1-CM2 des éditions Hatier (2011), qui n'exclut pas les gérondifs (y compris dans des exercices portant sur la «chronologie des évènements » : p. 65, ex. 7), ménage une séance spécifique sur le participe présent (p. 81, pour les CM2), en invitant notamment les élèves à rapprocher cette forme des adjectifs verbaux en -ant (ainsi «brillant» ou «suivant», que les auteurs appellent néanmoins des «noms»).

Cet apport est quelquefois significatif et témoigne dans certains cas d'une réflexion que nous appellerons «intégrative» de toutes les formes du système verbal. Cela étant, la contribution des manuels scolaires 
dans la compréhension de ce qui fait la participation des formes non fléchies du verbe à l'expression du temps est évidemment variable. Ces divergences étant plus ou moins marquées, il convient de les envisager dans le détail des séances et des exercices, comme nous nous y employons ci-après.

\section{Brève analyse comparative}

L'approche comparative menée ici auprès d'un échantillon de quatre manuels, dans lesquels sont variablement mises en ouvre des approches des formes verbales non fléchies, apporte une illustration plus précise des tendances qui prévalent sur ces questions. Ci-après nous relevons, dans ces ouvrages (parus en 2009, 2010 et 2012), les principales entrées pédagogiques qui s'appuient diversement sur les formes en question, lors de mises au point, d'exercices ou de prolongements à l'occasion desquels celles-ci sont abordées soit pour elles-mêmes, soit pour ce qu'elles permettent d'exercer ou de faire reconnaitre. Nous énumérons ci-dessous les activités qui correspondent à la pratique effective qui en est proposée, en indiquant les formes verbales concernées ainsi que la pagination, ce qui nous conduira à définir les manières dont les manuels relient les participes, l'infinitif et le gérondif soit à l'expression du temps, soit à d'(autres) éléments sur lesquels ils prennent appui. En voici la présentation tabulaire : 


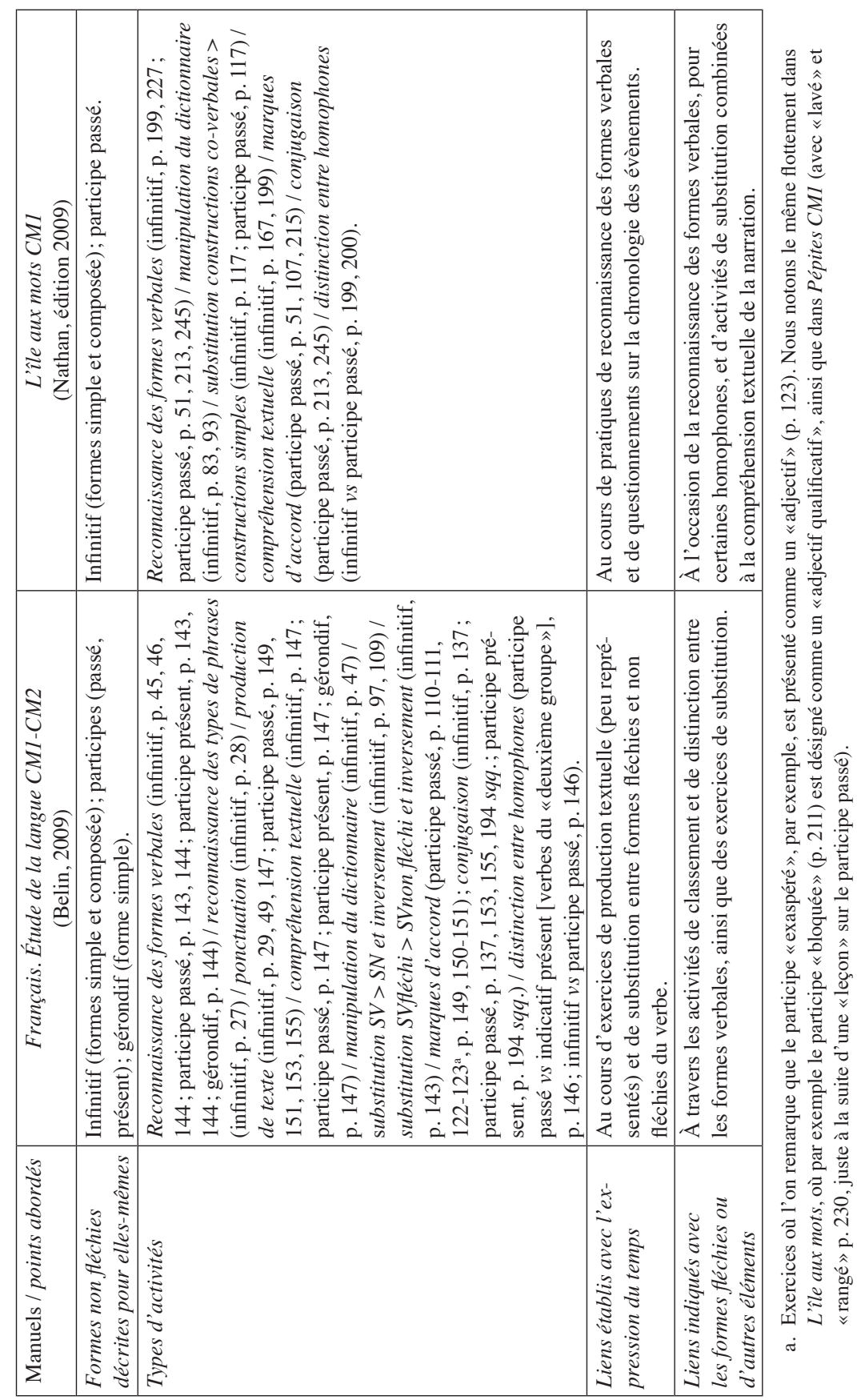




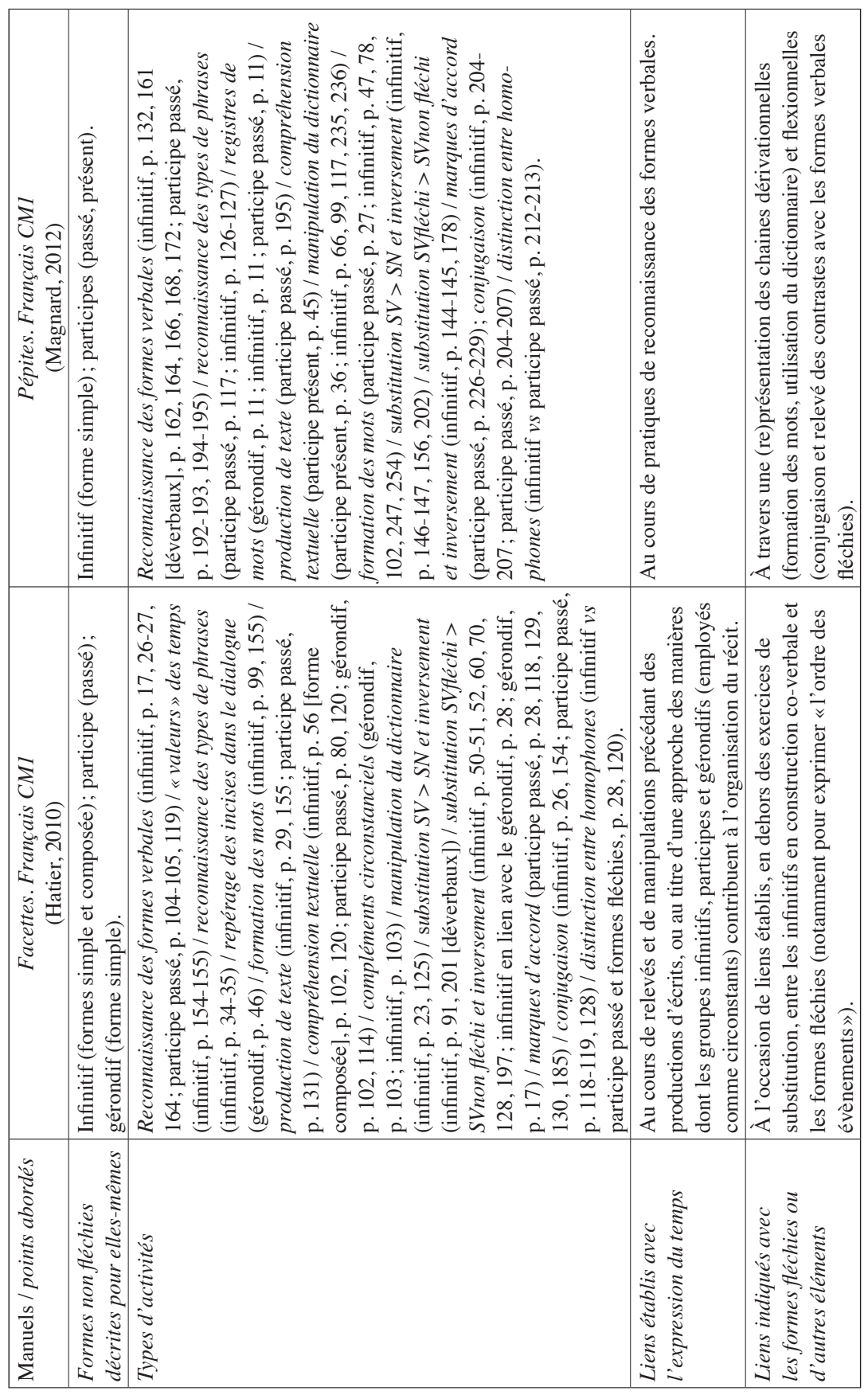


Dans les manuels ici pris en compte, les formes verbales non fléchies, quand elles sont abordées pour elles-mêmes, interviennent dans des séances qui leur sont au moins en partie consacrées ou, par ailleurs, dans des activités au cours desquelles leur emploi est envisagé comme un véritable objet de ou pour l'apprentissage. Dans les autres cas, les participes et l'infinitif en particulier apparaissent indirectement comme des contenus à enseigner, a priori non déductifs. On remarque par exemple, dans Français CM1-CM2 (2009), un exercice (15, p. 27) invitant les élèves à relever les «phrases injonctives» dans un extrait de Pierre et Camille de Musset, qui contient la présence d'une construction absolue de l'infinitif («-Vous mettre sur mes épaules»), et ce en réponse à une question posée avec un verbe fléchi. De même apparait une interrogation dans un exercice page 28 («et pour quoi faire?») où il s'agit de rétablir la ponctuation en lien avec les répliques précédente et suivante, toutes deux avec verbe fléchi (l'exclamation est très peu envisagée dans ce type d'approche, même si nous remarquons par exemple un «Ouf, nous voici débarrassés de ce tueur!» dans Pépites $C M 1$ [p. 117], avec un déverbal combiné à un participe passé dans une phrase d'exercice). Les manuels diversifient plutôt les «manipulations» de phrases représentatives, reprises de textes ou reformulées, à partir notamment de récits variés. Ainsi, Facettes CMI fait manipuler des phrases telles que «ce jour-là, Ulysse (réaliser) son rêve en accostant sur Ithaque» (p. 28), en combinant des gérondifs ou des infinitifs avec des formes fléchies à reconstituer. De même, Pépites CMI inclut-il plusieurs exercices demandant aux élèves de «justifier la terminaison des formes verbales en indiquant le mot qui [...] donne un signal» (p. 213), avec des groupes verbaux tels que «je viens de voir son frère» ou «le volcan va cracher de la lave $»^{6}$. Concernant là encore le recours à des constructions co-verbales, nous remarquons dans L'île aux mots CM1, et pour ne prendre que cet exemple, une activité d'observation (p. 167) qui soumet aux élèves un extrait de la Chèvre de Monsieur Seguin (A. Daudet) contenant, entre autres, l'énoncé «il se mit à rire méchamment». La question posée suggère alors discrètement la possibilité, pour ces constructions périphrastiques avec l'infinitif, de contribuer à l'expression du temps :

Pour chaque phrase avec plusieurs verbes, indique si les actions se déroulent en même temps ou l'une après l'autre. (Q.4)

6. Les italiques dans les exemples (de même par la suite) sont de nous. 
Dans une perspective didactique similaire, Facettes $C M 1$, à la suite de la phrase «après avoir été secouru par son cousin, Martin rentre dans sa chambre» (p. 56), invite les élèves, dans le cadre d'une séance où il est question de «succession» et de «simultanéité», à :

Releve[r] les mots et les expressions de temps qui permettent de suivre la succession des évènements ${ }^{7}$.

Ces consignes permettent de positionner la réflexion des élèves sur les rapports qui s'établissent, dans les textes, entre les formes verbales et éventuellement d'autres éléments, autant de rapports qui sont propres à éclaircir les temporalités sur lesquelles s'appuient la construction narrative et des informations comme l'antériorité ( $v s$ simultanéité) et l'accompli ( $v s$ en voie d'accomplissement), parmi lesquelles s'organisent des complémentarités.

Pour ce qui relève des séances spécifiques, nous retiendrons surtout que l'ouvrage Français CM1-CM2 ménage une rubrique intitulée «Infinitif et participe» (p. 142 sqq.), dans laquelle l'infinitif, qui «donne son nom au verbe» (p. 142) est aussi présenté comme une forme aux significations temporelles variables. Dans cette rubrique apparait en outre l'existence de «verbes dont la terminaison est -ant», et qui sont indiqués comme correspondant à «des participes présents ou des gérondifs ». Outre la substitution de formes verbales fléchies en infinitifs (p. 143) ou encore des manipulations conduisant les élèves à remplacer les infinitifs par des participes passés et présents (p. 143, 145), il est en effet brièvement question du gérondif, lequel est néanmoins décrit par le schème «en + participe présent» à la page 144. La plupart des supports d'activités sont des extraits de textes au passé, avec des prolongements en termes de «vocabulaire» où la distinction entre participe présent et adjectif verbal («fatigante», «brillantes»), les cas de nominalisation («un passant») ainsi que de grammaticalisation («pendant le dîner») interviennent là aussi explicitement à l'intérieur de phrases représentatives $^{8}$. L'infinitif, de son côté, sert à de multiples reprises pour désigner le verbe en dehors de toute marque flexionnelle, pour ainsi dire dans

7. Il sera question p. 102, dans ce manuel, de relever des compléments circonstanciels dans un récit, parmi lesquels «sans se retourner» et «en se cachant dans la nuit» (à la page suivante, les élèves ont à relever, parmi d'autres circonstants, «sans faire de bruit» et «en découvrant une piste»).

8. Pour les adjectifs verbaux, certains manuels prennent le parti de parler plus généralement d' «adjectifs », comme Pépites CM1 (2012), lequel reprend 
sa version dictionnairique, telle qu'y recourent la plupart des manuels (voir Roubaud, 1998). Pour autant, certains exercices, à l'appui de textualités diverses, demandent à l'élève de «choisir» entre la forme non fléchie et les formes fléchies (le présent de l'indicatif dans un extrait de texte descriptif, l'imparfait dans un texte narratif, etc.).

Comme indiqué supra, l'infinitif est évidemment la forme la plus représentée, à travers notamment les exercices de substitution (des formes non fléchies aux formes fléchies et vice versa), dans des rubriques qui hésitent le plus souvent à l'inscrire dans un questionnement sur le temps exprimé en dehors de quelques périphrases. Cette contradiction se rencontre jusque dans les activités de repérage : ainsi Pépites CM1, au moment de faire «repére[r] les verbes», par les élèves, afin d' «explique[r] leur accord» (p. 233, ex. 13), s'appuie-t-il sur un texte documentaire de 8 lignes en colonne où l'on trouve rien moins que les expressions «pour protéger leurs chèvres», «pour éviter cela» et «retournent chasser», avec des infinitifs qui, semble-t-il, ne sont pas envisagés comme des verbes. Dans L'île aux mots au contraire, alors que la première séance sur l'infinitif débute sur une reconnaissance de la forme en complémentarité avec les formes verbales fléchies (dans un récit au passé), les élèves sont immédiatement sensibilisés à l'existence de constructions prépositives («pour revoir le bouquetin»), coverbales («ils allaient abandonner», «ils se sont mis à observer»), et de constructions subordinatives («nous l'avons vu se mettre à sauter»), parmi lesquelles le rattachement entre les formes fléchies et l'infinitif est contextualisé afin de clarifier leurs positionnements temporels respectifs.

Ces exemples sont évidemment sommaires, mais ils témoignent de choix didactiques contrastés, suivant la place que les auteurs concèdent aux formes verbales non fléchies et à leur contribution à l'expression du temps. Ces choix sont d'autant plus délicats à caractériser qu'en la matière, la quantité des exercices ne garantit pas forcément la qualité des questionnements soumis aux élèves. À l'inverse, quand une réflexion didactique de fond existe sur cette question de l'expression temporelle des formes verbales non fléchies, les auteurs semblent hésiter à la concrétiser et à se saisir pleinement de l'enjeu que les Programmes désignent à travers non pas uniquement l'usage et le réemploi, mais la «compréhension» du système verbal dans son ensemble.

notamment les mots «effrayante» (p. 17), «croustillant» (p. 152) et «attirants» (p. 231). 


\section{En conclusion}

Concernant les exercices pratiqués à l'appui des manuels sur l'approche des temporalités représentées dans les textes, il nous semble opportun d'insister sur le gain que représente un retour sur la complémentarité existant entre les formes «conjuguées» et les formes «non conjuguées» du verbe. Dans la mesure où s'exerce généralement un rattachement temporel concret des unes aux autres, ces faits permettent d'envisager leurs relations à travers non pas uniquement les paradigmes flexionnels, mais également des temporalités susceptibles d'être discursivement déduites par les élèves. À ce titre, rappelons qu'aussitôt prédiquée, la forme verbale non fléchie participe forcément de l'expression du temps, tandis qu'en termes de constructions, soit ces formes sont susceptibles de se construire grammaticalement et d'exprimer discursivement le temps par elles-mêmes (ce qui est principalement le cas de l'infinitif et du participe présent), soit elles apparaissent comme forcément rattachées à d'autres éléments discursifs (généralement des verbes fléchis).

L'apport des manuels scolaires témoigne, dans ce domaine, de nombreuses réticences, mais aussi de quelques initiatives. Le principal point d'achoppement pour l'analyse qu'on peut en faire est la dilution de ces contenus d'apprentissage parmi les rubriques dans lesquelles les représentations de l'infinitif et des participes, notamment, sont malléables et peuvent aboutir quelquefois à l'inverse de leurs objectifs. Il convient, de ce point de vue, d'envisager les manières dont les manuels se soustraient en partie à la chronologie passé-présent-futur pour ouvrir les perspectives vers d'autres visions de la temporalité. Une analyse comparée des repères linguistiques qui sont soumis aux élèves dans les manuels et ceux relevés au cours des pratiques effectives d'enseignement permettrait, sans doute, de mettre à jour leurs dissemblances, mais aussi leurs complémentarités pour ce qui touche aux formes verbales non fléchies. L'enjeu est d'autant plus important que celles-ci, de par leur absence de flexion, sont en elles-mêmes une invitation à reformuler les représentations du temps verbal à travers des traits mésestimés tels que l'extension temporelle, la concomitance ou la momentanéité, parmi bien d'autres possibilités. 


\section{RÉFÉRENCES BIBLIOGRAPHIQUES}

Arrivé, M., Gadet, F. \& Galmiche, M. (1986). Grammaire d'aujourd'hui. Guide alphabétique de linguistique française. Paris : Flammarion.

Bonami, O. \& Boyé, G. (2008). Quels verbes sont réguliers en français? Dans J. Durand, B. Habert \& B. Laks (dir.), Actes du Premier congrès mondial de linguistique française (p. 1511-1523). Paris : ILF.

Charaudeau, P. (1992). Grammaire du sens et de l'expression. Paris : Hachette.

Cogis, D. (2005). Pour enseigner et apprendre l'orthographe. Paris : Delagrave.

David, J. \& Renvoisé, C. (2010). La Morphologie verbale : repérer les complexités et les régularités. Synergie France, 6, 61-75.

Deulofeu, M. J. (2009). Pour une linguistique du rattachement. Dans D. Apothéloz, B. Combettes \& F. Neveu (dir.), Les Linguistiques du détachement (p. 229-250). Berne : Peter Lang.

Deulofeu, M. J. (2010). Portée sémantique et rattachement syntaxique vers l'amont des constituants périphériques non phrastiques en français parlé. Dans les Actes du XXV Congrès international de linguistique et philologie romanes d'Innsbruck (p. 359-370). Berlin : De Gruyter.

Dourojeanni, D. \& Quet, F. (2007). Problèmes de grammaire pour le cycle 3. Enseigner la langue par l'observation, la réflexion et le débat. Paris : Hatier Mosaïque.

Dressler, W. (1997). On Productivity and Potentiality in Inflectionnal Morphology. Dans CLASnet Working Papers (Vol. 7, p. 3-22). Montréal : CLASnet.

Eluerd, R. (2011). Grammaire descriptive de la langue française. Paris : Armand Colin, coll. «Cursus».

Germain, C. \& SÉguin, H. (1998). Le Point sur la grammaire. Paris : CLE International.

Gettrup, H. (1977). Le Gérondif, le participe présent et la notion de repère temporel. Revue Romane, 12 (2), 210-271.

Halmøy, О. (2003). Le Gérondif en français. Paris : Ophrys.

Havu, E. (2009). Prédications secondes adjectivantes : l'interprétation des participes présents adjoints. Dans I. Evrard, M. Pierrard, L. Rosier \& D. Van Raemdonck (dir.), Représentations du sens linguistique 3 (p. 89-104). Bruxelles : De Boeck.

KLeIBer, G. (2007). La Question temporelle du gérondif : simultanéité ou non? Travaux Linguistiques du Cerlico, 20, 109-123.

LAZARD, G. (1985). Les variations d'actance et leurs corrélats. Actances, $1,5-39$. 
LusetTi, M. (2008). Le verbe pour commencer la grammaire au CE1. Recherches (L'Enseignement de la langue), 48, 105-135.

Martin, R. (1971). Temps et aspect. Paris : Klincksieck. Masseron, C. (1998). Présentation. Pratiques, 100, 3-6.

Meleuc, S. (2005). Écrire le verbe : propriétés linguistiques et didactisation. Dans C. Vaguer \& D. Leeman (dir.), De la Langue au texte (Le Verbe dans tous ses états, Vol. 2, p. 49-66). Namur: PUN.

Meleuc, S. \& Fauchart, N. (1999). Didactique de la conjugaison. Le Verbe «autrement». Toulouse : Bertrand-Lacoste, CRDP Midi-Pyrénées.

Muller, C. (2006). Participe présent, conjonction et construction du sujet. Dans F. Lambert, C. Moreau \& J. Albrespit (dir.), Les Formes non finies du verbe (Vol. 2, p. 19-36). Travaux linguistiques du CerLiCO, 19.

Riegel, M., Pellat, J.-C. \& Rioul, R. (1999 [1994]). Grammaire méthodique du français. Paris : PUF.

Roubaud, M.-N. (1998). L'Infinitif du verbe. Pratiques, 100, 7-22.

Sautot, J.-P. \& Lepoire-Duc, S. (2009). Le « socle commun de connaissances» fait-il norme pour l'enseignant? Le cas de la grammaire à l'école élémentaire. Dans D. Dubois-Marcoin \& C. Tauveron (dir.), Français, littérature, socle commun. Quelle culture pour les élèves? Quelle professionnalité pour les enseignants? (p. 215-228). Lyon : INRP.

Torterat, F. (2012a). L'infinitif et le gérondif, une désillusion pour la conjugaison? Dans P.-Y. Dufeu \& S. Oueslati (dir.), L'Illusion taxinomique (p. 36-48). Tunis, ISSH éditions.

Torterat, F. (2012b). Les Participes, l'Infinitif et le Gérondif, entre scalarité et rattachement (+/- local). Studii de Linguistică, 2, 169-210.

TournadRe, N. (2004). Typologie des aspects verbaux et intégration à une théorie du TAM. Bulletin de la Société de linguistique de Paris, XCIX (1), 36-48.

Van Raemdonck, D. \& Detaille, M. (2011). Le sens grammatical. Référentiel à l'usage des enseignants. Bruxelles : PIE Peter Lang. 